\title{
Latest Developments in FIB Technology and Applications
}

Lucille A. Giannuzzi

FEI Company, 5350 NE Dawson Creek Drive, Hillsboro, OR 97124

$\mathrm{Ga}^{+}$liquid metal ion source (LMIS) focused ion beam (FIB) technology has improved drastically over the past decade or so. As an example, research performed over 20 years ago using FIB for transmission electron microscopy (TEM) specimen preparation used FIB columns with $\sim 50-100$ $\mathrm{nm}$ resolution [1,2]. Today, $\mathrm{Ga}^{+} \mathrm{FIB}$ columns operate up to $20 \mathrm{x}$ better at $\leq 5 \mathrm{~nm}$ resolution. The current density of the ion beam has also improved yielding faster milling rates and precise milling acuity. The upper limit of available beam current has increased over the years, with $\mathrm{Ga}^{+} \mathrm{FIB}$ columns routinely offering $>50 \mathrm{nA}$. In addition, low energy $\mathrm{Ga}^{+} \mathrm{FIB}$ specimen preparation techniques yield less implantation damage necessary for qualitative high resolution TEM microscopy [3], improved electron backscattered diffraction patterns [4], and reduced ion mixing and damage for atom probe tomography [5].

Latest improvements to $\mathrm{Ga}^{+} \mathrm{FIB}$ columns include time of flight (TOF) corrections for the ions accelerating through the column. TOF corrections match the ion velocity through the column with the timing of the electrostatic scanning and blanking signals. TOF corrections are particularly useful at low beam energy and as the beam dwell times get shorter and shorter (i.e., faster beam blanking scan times). FIG. 1 shows improved pattern accurary using a $5 \mathrm{keV} \mathrm{Ga}^{+}$FIB that has been fully TOF corrected.

In addition to LMIS sources, gas field ion sources (GFIS) [6,7] and inductively coupled plasma (ICP) sources [8] have also been introduced into FIB columns. Light ions have been incorporated into GFIS FIBs primarily for unique imaging capabilities [9]. ICP sources yield worse ultimate resolution than LMIS FIBs within the low current regime, but greatly improved resolution in the high current regime [8]. Thus, the ICP FIB allows for faster removal rates of large volumes. As an example, a comparison of milling capabilities between the LMIS Ga${ }^{+}$FIB and the ICP Xe ${ }^{+}$FIB are shown in FIG. 2. The removal rates for the ICP FIB is $~ 20-100$ times greater than that for the LMIS FIB. The increased milling rates are due to the combination of the heavier ion which yields higher sputter yields (i.e., $\mathrm{Xe}^{+}$vs $\mathrm{Ga}^{+}$) plus the larger beam currents that are available with the ICP FIB [10].

\section{References}

[1] E.C.G. Kirk et al., Inst. Phys. Conf. Ser. 100 (1989) 501.

[2] Kyung-ho Park, Mat. Res. Soc. Symp. Proc. 199 (1990) 271.

[3] Lucille A. Giannuzzi et al., Microsc Microanal 11(Suppl 2) (2005) 828.

[4] J.R. Michael and Lucille A. Giannuzzi, Microsc Microanal 13(Suppl 2), (2007) 926CD.

[5] K. Thompson et al., Ultramicroscopy 107 (2007) 131.

[6] X. Liu and J. Orloff, Adv. in Imag. and Elect. Phys., 138 (2005) 147.

[7] X. Liu and J. Orloff, J. Vac. Sci. Tech. B 23 (2005) 2816.

[8] N.S. Smith et al., J. Vac. Sci. Technol. B 24(6) (2006) 2902.

[9] J. Notte et al., Microsc Microanal 12(Suppl 2) (2006) 126.

[10] Sean Kellogg and Chad Rue at FEI provided the ICP FIB images. Tomas Nemecek and Bohus Seda at FEI provided the TOF FIB images. Thanks to Richard Young, FEI, for helpful comments. 

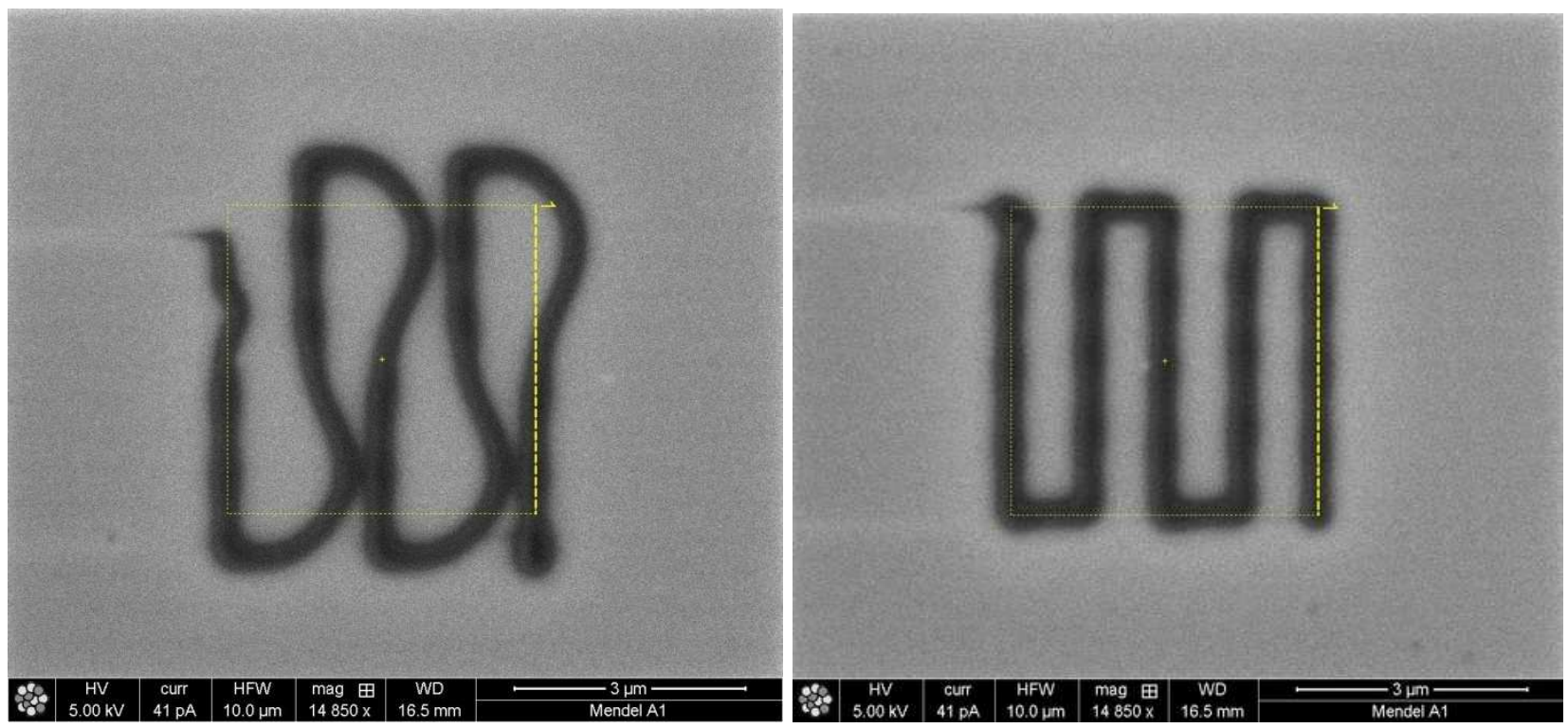

FIG.1. Time of flight (TOF) corrections for patterning with $5 \mathrm{keV} \mathrm{Ga}^{+}$ion beam, $41 \mathrm{pA}$ beam current, $300 \mathrm{~ns}$ dwell time. (left) with partial TOF correction, (right) with full TOF correction.
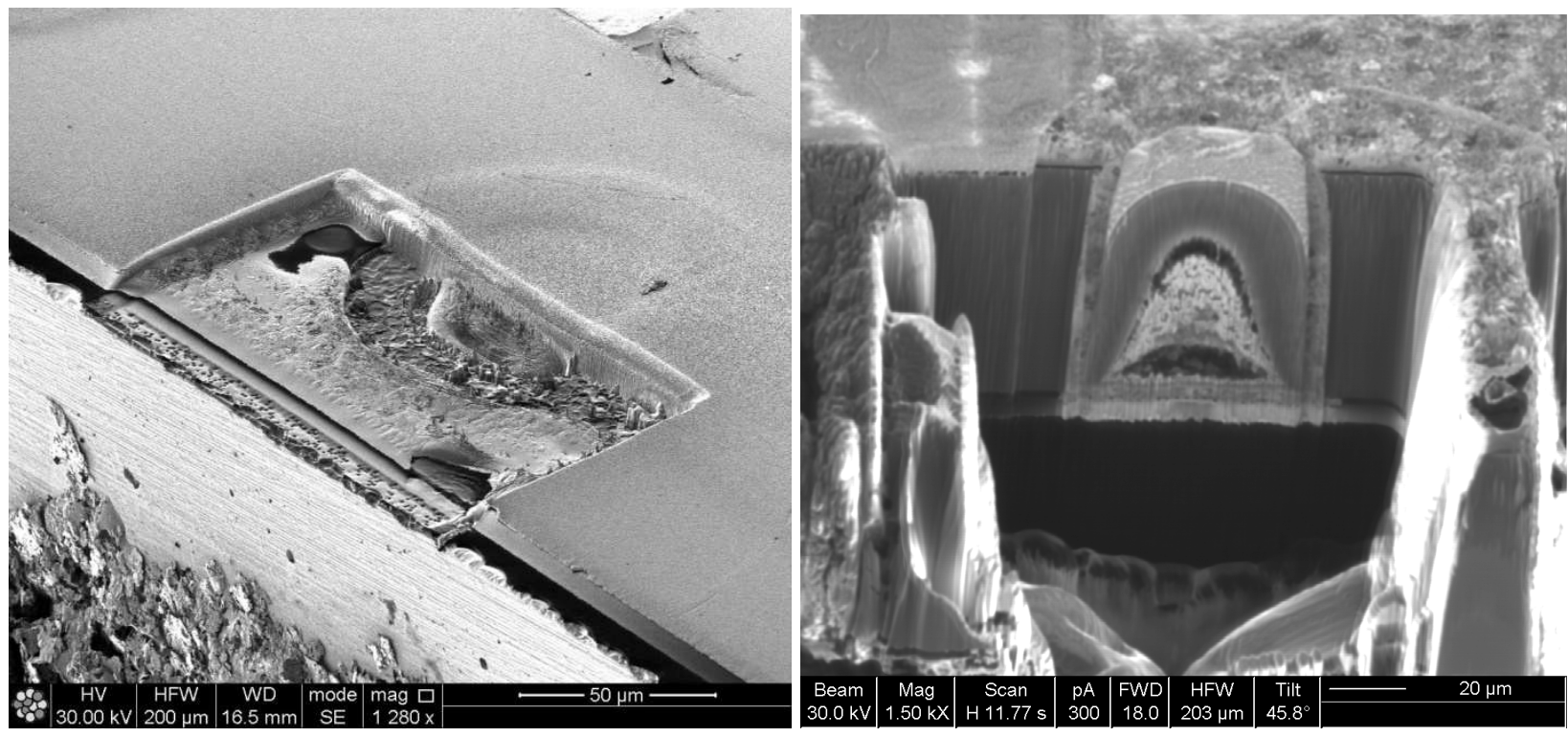

FIG.2. $\mathrm{Ga}^{+}$FIB images of $\mathrm{Ga}^{+}$LMIS FIB vs. $\mathrm{Xe}^{+}$ICP FIB cross sectioning capabilities. The defined pattern size is $\sim 100 \mu \mathrm{m}$ by $60 \mu \mathrm{m}$. Left image shows $60 \mathrm{~min} \mathrm{Ga}^{+} \mathrm{FIB}$ at $21 \mathrm{nA}$. Right image shows $30 \mathrm{~min} \mathrm{Xe}^{+} \mathrm{ICP}$ FIB at $1 \mu \mathrm{A}$. (sample courtesy of ST-Ericson) 